\title{
Parameter Identification of a Three-dimensional Flexible Ring-based Model of a Tire Using Experimental Modal Analysis
}

\author{
Masami Matsubara ${ }^{1)}$ Shozo Kawamura ${ }^{1)}$ \\ 1) Toyohashi University of Technology \\ 1-1 Hibarigaoka Tempaku-cho, Toyohashi, Aichi, 441-8580, Japan (E-mail: matsubara@me.tus.jp)
}

Received on June 8, 2018

\begin{abstract}
A flexible ring-based model is simple, comprehensive, and accurate, and has been most frequently adopted in tire models with a low degree-of-freedom. Despite the importance of parameter identification techniques, the literature pertaining to the discussion of these techniques is insufficient. This paper presents a novel parameter identification method based on a three-dimensional flexible ring-based model for the tires of a passenger car using experimental modal analysis. This method is able to identify modal parameters by using experimental modal analysis and by using the model to calculate the model parameters for comparison with the modal parameters. The experimental modal analysis entailed the use of a method based on an iterative simultaneous equation and confirmed that it was possible to estimate the modal parameters with high accuracy. The recalculated results using the model parameters showed good correlation with the experimental results.
\end{abstract}

KEY WORDS: Vibration, noise, and ride comfort, brake/tire, test and analysis technology [B3]

\section{Introduction}

Tires are important automotive parts that are in contact with the road surface and they have a dominant influence on the steering stability and noise, vibration, and harshness (NVH) performance. The development of a vehicle involves numerical analysis of the vehicle dynamics, and simplified tire models are used to reduce the amount of calculation and model construction $\operatorname{cost}^{(1)}$. Additionally, it is also used to obtain a simple estimation of the tire design parameters ${ }^{(2)}$. Earlier studies proposed a threedimensional flexible ring-based model ${ }^{(3)(4)}$. This model can capture tread vibrations below $150 \mathrm{~Hz}$. Unlike most studies that used flexible ring models ${ }^{(5)-(7)}$ and mainly discussed radial and circumferential vibration, this model can also analyze lateral vibration $^{(4)}$ and is able to take into account the wheel model ${ }^{(8)}$. Therefore, it is also possible to estimate the sidewall stiffness as tire design parameter ${ }^{(9)}$. In general, the accuracy of parameter identification is directly related to the accuracy of the analysis. Hence, the discussion of parameter identification techniques is important, yet insufficient research has been reported. Previous studies have presented approaches using the natural frequency, which is one of the modal parameters ${ }^{(3)}$ or using the minimum value problem of the residue between the experimental frequency response function (FRF) and the analytical value ${ }^{(4)}$. When the FRF is used, if it is possible to identify modal parameters with high accuracy from experiments, the identification accuracy of the model parameters can be considered to improve by using modal parameters.

This paper presents a novel model parameter identification method based on a three-dimensional flexible ring-based model using experimental modal analysis (EMA). The model parameter of a three-dimensional flexible ring-based model includes values related to the model dimension, inflation pressure, tread and sidewall stiffness, and damping characteristics. First, the FRF in the model is derived, and the modal parameters are then extracted from the FRF. Second, experimental modal analysis is performed on an actual tire. A subsequent comparison of the modal parameters between the analysis and experiment enables the model parameters to be identified. Thus, our method, which uses a linear fit method using the real and imaginary parts of FRF, is designed to identify mode characteristics with higher accuracy than conventional methods ${ }^{(10)}$. Finally, the experimental data is compared with the parameters obtained with the FRF, after which the model parameters were used to recalculate the results. These results are presented in this paper and indicate the validity of the proposed method.

\section{Three-dimensional flexible ring-based model}

\subsection{Outline of model ${ }^{(3)(4)}$}

Fig.1(a)-(d) show an outline of the three-dimensional flexible ring-based model. The following tire modeling assumes a nonloaded and non-rolling condition. The proposed tire model is a three-dimensional flexible ring model in which a thin cylindrical shell ring, springs, and a cylinder represent the tire tread including the belt, sidewall, and wheel, respectively (Fig.1(a)). Unlike most proposed ring models, this model uses two springs to express the lateral, circumferential, and radial stiffness of the sidewall. The springs connect the edges of the tire tread to the edges of the wheel. The cylinder is a rigid body owing to its high stiffness relative to the tire and to being fixed in space. Fig.1(b) indicates that location of the tread-ring element described using a cylindrical coordinate system $(y, \theta, r)$ in which $r=R$ is considered the neutral plane of the tread ring and the vibrational displacement of an arbitrary point on the neural plane with respect to $(y, \theta, r)$ is $(u, v, w)$ in Fig.1(c). The parameters of the presented model, 
shown in Fig.1(d), are as follows: for the tread ring, the radius is $R$, thickness $b$, width $2 l$, second moment of area $I$, modulus of longitudinal elasticity $E$, Poisson's ratio $v$, tension $S_{0}$, and mass density $\rho$; for the sidewall, the lateral, circumferential, and radial sidewall stiffness values are $K_{y}, K_{\theta}$, and $K_{r}$, respectively. In this study, it is necessary to consider the damping properties when deriving the motion equation. Dynamic properties are mostly described in terms of complex stiffness related to the force of restitution as $S_{0}, K_{y}, K_{\theta}$, and $K_{r}$. The structural damping model is expressed as ${ }^{(4)}$

$S_{0}^{*}=\left(1+\mathrm{j} \eta_{s}\right) S_{0}$

$K_{y}^{*}=\left(1+\mathrm{j} \eta_{y}\right) K_{y}$

$K_{\theta}^{*}=\left(1+\mathrm{j} \eta_{\theta}\right) K_{\theta}$

$K_{r}^{*}=\left(1+\mathrm{j} \eta_{r}\right) K_{r}^{*}$

where $\mathrm{j}$ is a purely imaginary number, $S_{0}{ }^{*}, K_{y}{ }^{*}, K_{\theta}{ }^{*}$, and $K_{r}{ }^{*}$ are complex parameters, and $\eta_{s}, \eta_{y}, \eta_{\theta}$, and $\eta_{r}$ are structural damping coefficients related to the tension $S_{0}$, and the radial, circumferential, and lateral sidewall stiffness values are $K_{y}, K_{\theta}$, and $K_{r}$. If the potential energy $U$ is derived using $S_{0}{ }^{*}, K_{y}{ }^{*}, K_{\theta}{ }^{*}$, and $K_{r}^{*}, U$ includes the purely potential energy and dissipation energy due to damping. Given the thinness of the tread compared with the full tread radius, the lateral strain of the tread ring, circumferential strain of the tread ring, and shear strain of the neutral plane are assumed to be $0^{(11)}$. In addition, inextensional tread deformation due to vibration is assumed ${ }^{(12)}$. The aforementioned strains are expressed as

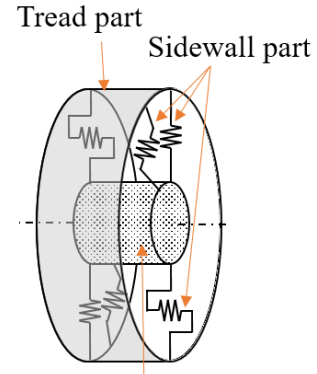

Wheel part

(a) Outline of model

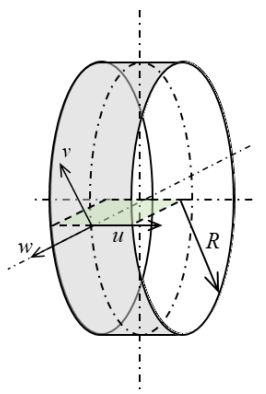

(c) Coordinate system on the neural plate

Fig.1 Outline of a three-dimensional flexible ring-based for passenger car's tires

$\varepsilon_{y}=\frac{\partial u}{\partial y}=0$

$\varepsilon_{\theta}=\frac{\partial v}{R \partial \theta}+\frac{w}{R}=0$

$\gamma_{y \theta}=\frac{\partial u}{R \partial \theta}+\frac{\partial v}{\partial y}=0$

In this study, we focused on the tire vibration characteristics under non-loaded and non-rolling conditions. Hence, potential energy is not stored in the tread ring in the bending and torsion in modes excited below $200 \mathrm{~Hz}$ because of the assumption of an inextensional tread ring $^{(7)}$. Cooley and Parker presented the comparison of vibration of a rotating ring using a fully extensible ring model and its corresponding inextensible ring model ${ }^{(13)}$. According to their paper, the two models agree in terms of the lower natural frequencies at bending-dominated modes at low rotation speeds. Thus, the parameters $E, I$, and $v$ are not considered under non-loaded and non-rolling conditions. From the above, we can calculate the mechanical energy by defining eigenfunctions from Eqs.(4), and the Lagrangian equation of motion can be obtained. Samples of the mode shape obtained by eigenvalue analysis are shown in Fig.2.

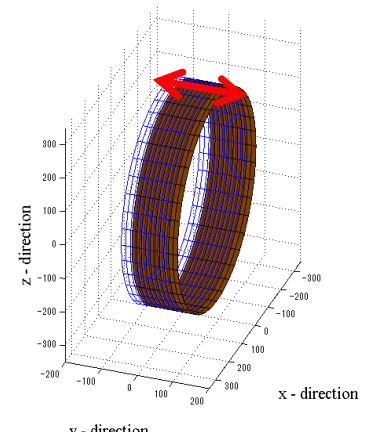

$\mathrm{y}$ - direction

(a) Lateral translational mode

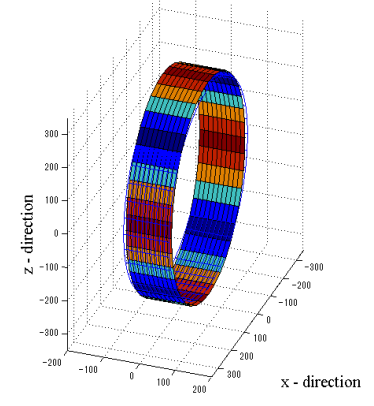

$$
y \text { - direction }
$$

(c) 2nd circumferential mode

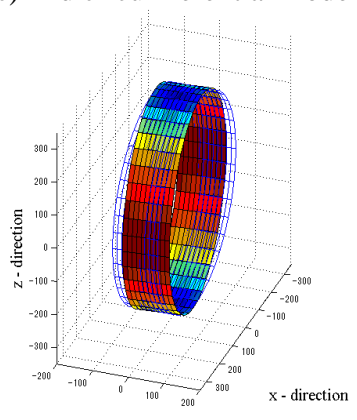

$y$ - direction

(e) 1st lateral bending mode

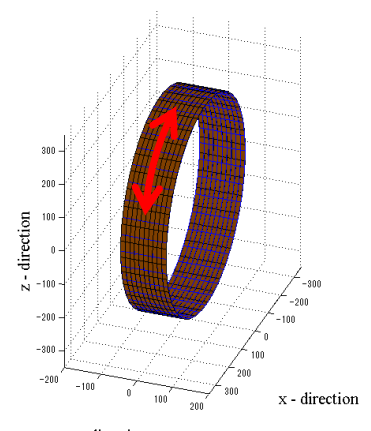

(b) Torsional mode

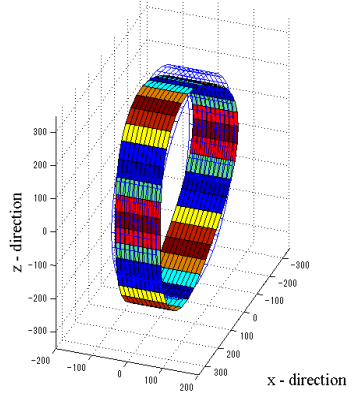

$$
y \text { - direction }
$$

(d) 3rd circumferential mode

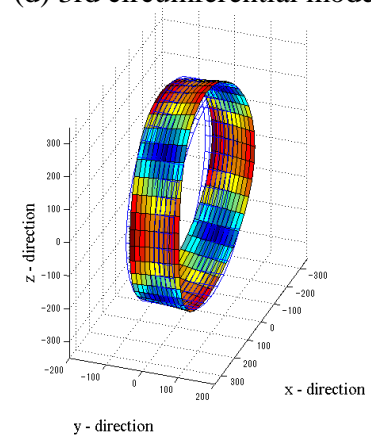

(f) 2nd lateral bending mode

Fig.2 Samples of mode shapes

2.2. Frequency response function on the tread part 
This section presents the proposed method for identifying the model parameters from EMA. Modal parameters are estimated by frequency-domain modal analysis, and the model parameters are calculated by comparing them with the modal parameters calculated from the model. Thus, we need to derive the FRF including the model parameters. Because the aim is to improve the identification accuracy by using experimental data with a large contribution of each of the model parameters, it is desirable that the derived FRF includes the contribution of mode shapes shown in Fig.2. Thus, the excitation position, at which the tread of the tire vibrates in the lateral, circumferential, and radial direction, and the response position, at which it can be grasped, are determined. In this study, we derived the FRF between the input at the tread edge in the lateral direction, the input at the tread center in the circumferential and radial direction, and the response at the tread center. The formulas of the accelerance FRFs are as follows:

$$
\begin{aligned}
& H_{u}=\frac{(2 \pi f)^{2}}{\left\{K_{\mathrm{tra}}-(2 \pi f)^{2} M_{\mathrm{tra}}\right\}}+\sum_{m=1}^{M} \frac{R^{2}(2 \pi f)^{2}}{n^{2}\left\{K_{\mathrm{b}, m}-(2 \pi f)^{2} M_{\mathrm{b}, m}\right\}} \\
& H_{v}=\frac{(2 \pi f)^{2}}{\left\{K_{\mathrm{tor}}-(2 \pi f)^{2} M_{\mathrm{tor}}\right\}}+\sum_{n=1}^{N} \frac{(2 \pi f)^{2}}{\left\{K_{\mathrm{r}, n}-(2 \pi f)^{2} M_{\mathrm{r}, n}\right\}} \\
& \left.H_{w}=\sum_{n=1}^{N} \frac{n^{2}(2 \pi f)^{2}}{\left\{K_{\mathrm{r}, n}-(2 \pi f)^{2} M_{\mathrm{r}, n}\right.}\right\}
\end{aligned}
$$

where $f$ is an arbitrary frequency, and $m$ and $n$ are natural numbers and mode numbers of lateral bending and radial modes, respectively. $M$ and $N$ are the maximum mode number of lateral bending and radial modes, respectively. The mass and stiffness terms are as follows:

$$
\begin{aligned}
& M_{\mathrm{tra}}=4 \pi R \rho b l, K_{\mathrm{tra}}=4 \pi R K_{y}^{*} \\
& M_{\mathrm{tor}}=4 \pi R \rho b l, K_{\mathrm{tor}}=4 \pi R K_{\theta}^{*} \\
& M_{\mathrm{r}, n}=2 \pi R \rho b l\left(n^{2}+1\right) \\
& K_{\mathrm{r}, n}=2 \pi R\left[\frac{S_{0}^{*}}{R^{2}} \ln ^{2}\left(n^{2}-1\right)+K_{\theta}^{*}+n^{2} K_{r}^{*}\right] \\
& M_{\mathrm{b}, m}=2 \pi R \rho b l\left(\frac{R^{2}}{n^{2}}+\frac{l^{2}}{3}+\frac{l^{2}}{3} n^{2}\right) \\
& K_{\mathrm{b}, m}=2 \pi R\left[\frac{S_{0}^{*}}{3 R^{2}} l^{3} m^{2}\left(m^{2}-1\right)\right. \\
&\left.\quad+\frac{R^{2}}{m^{2}} K_{y}^{*}+l^{2} K_{\theta}^{*}+m^{2} l^{2} K_{r}^{*}\right]
\end{aligned}
$$

\section{Proposal of parameter identification}

\subsection{Extracting the modal parameters from analytical FRFs}

In the previous section, the theoretical formula of FRF is derived. Therefore, if the modal parameters can be defined from the theoretical formula, they can be compared with the modal parameters obtained from the experimental modal analysis (EMA). EMA is a technique to identify the modal parameters, namely the natural frequencies, damping characteristics, and residue related to the modal shapes and one of the key technologies in structural dynamics analysis ${ }^{(14)}$. We consider the vibrations of a system with $N_{p}$ degrees-of-freedom. The dynamic compliance $\operatorname{FRF} H_{r, e}(f)$ for an arbitrary frequency $f$ is described by the following equation.

$$
H_{r, e}(f)=\sum_{p=1}^{N_{p}} \frac{\frac{\phi_{r, p} \phi_{e, p}}{k_{p}}(2 \pi f)^{2}}{\left(1-\frac{f^{2}}{f_{n p}^{2}}\right)+j \eta_{p}}=\sum_{p=1}^{N_{p}} \frac{R_{p}(2 \pi f)^{2}}{\left(1-\frac{f^{2}}{f_{n p}^{2}}\right)+j \eta_{p}}
$$

with $n$ the number of modes, $r$ the number of response points, $e$ the number of excitation points, $k_{p}$ the $p$-th modal stiffness, $\eta_{p}$ the $p$-th modal damping ratio, $f_{p}$ the $p$-th natural frequency, $\phi_{r, p}$ and $\phi_{e, p}$ the mode components with the $p$-th mode of the $r$-th and $f$-th vector components. The parameters of Eq.(7) to be estimated in the dynamic system with modes are the natural frequency $f_{p}$, the structural damping ratio $\eta_{p}$, and the residual term $R_{p}$ as $\left(\phi_{r, p} \phi_{e, p}\right) / k_{p}$ The use of EMA enables these parameters to be identified. Then, Eqs. (3)-(5) are transformed such that these parameters can be compared with the result of Eq. (10).

$H_{u}=\frac{\frac{1}{\operatorname{Re}\left(K_{\mathrm{tra}}\right)}(2 \pi f)^{2}}{1-\frac{f^{2}}{f_{\mathrm{tra}}^{2}}+j \eta_{\mathrm{tra}}}+\sum_{m=1}^{M} \frac{\frac{R^{2}}{m^{2} \operatorname{Re}\left(K_{\mathrm{b}, m}\right)}(2 \pi f)^{2}}{1-\frac{f^{2}}{f_{\mathrm{b}, m}^{2}}+j \eta_{\mathrm{b}, m}}$

$H_{v}=\frac{\frac{1}{\operatorname{Re}\left(K_{\mathrm{tor}}\right)}(2 \pi f)^{2}}{1-\frac{f^{2}}{f_{\mathrm{tor}}^{2}}+j \eta_{\mathrm{tor}}}+\sum_{n=1}^{N} \frac{\frac{1}{\operatorname{Re}\left(K_{\mathrm{r}, n}\right)}(2 \pi f)^{2}}{1-\frac{f^{2}}{f_{\mathrm{r}, n}^{2}}+j \eta_{\mathrm{r}, n}}$

$H_{w}=\sum_{n=1}^{N} \frac{\frac{n^{2}}{\operatorname{Re}\left(K_{\mathrm{r}, n}\right)}(2 \pi f)^{2}}{1-\frac{f^{2}}{f_{\mathrm{r}, n}^{2}}+j \eta_{\mathrm{r}, n}}$

where $f_{\text {tra }}, f_{\text {tor }}, f_{\mathrm{r}, \mathrm{n}}$, and $f_{\mathrm{b}, \mathrm{m}}$ are the lateral translational, torsional, radial, and lateral bending modes, $\eta_{\mathrm{tra}}, \eta_{\mathrm{tor}}, \eta_{\mathrm{r}, \mathrm{n}}$, and $\eta_{\mathrm{b}, \mathrm{m}}$ are the modal damping ratios related to each of the modes and ratios of real part and imaginary part of equivalent stiffness $K_{\mathrm{tra}}, K_{\mathrm{tor}}, K_{\mathrm{r}, \mathrm{n}}$, and $K_{\mathrm{b}, \mathrm{m}}$. By conducting a vibration test to obtain the FRFs of Eqs. (8)-(10) and performing EMA, it is possible to calculate the value related to the model parameters.

\subsection{Experimental modal analysis}

The FRF is measured experimentally by performing hammering tests. Fig. 3 shows the test setup. The tire size is $195 / 65 R 15$, and a commercial tire was used under non-loaded and non-rolling conditions. The tire was fixed to an axle and inflated to the standard pressure. External forces were measured by an impact hammer and the response during acceleration was measured by an accelerometer. Especially, it is difficult to excite vibration in the circumferential direction of the tire. Thus, an aluminum block was placed on the tread by using adhesive and we excited vibration in the circumferential direction. The disadvantage of this method is that it adds extra mass and cannot excite the high-frequency range. However, in model parameter identification, it is only necessary to confirm the low-frequency mode component, hence there is no particular problem in this research. A frequency analyzer was used to perform the FRF calculation. The maximum frequency at which measurements were conducted was $400 \mathrm{~Hz}$, and the frequency resolution was $0.125 \mathrm{~Hz}$. Subsequently, the FRF is read into the numerical calculation software and the EMA is performed. We adapt a method based on a linear fit method using the real and imaginary 


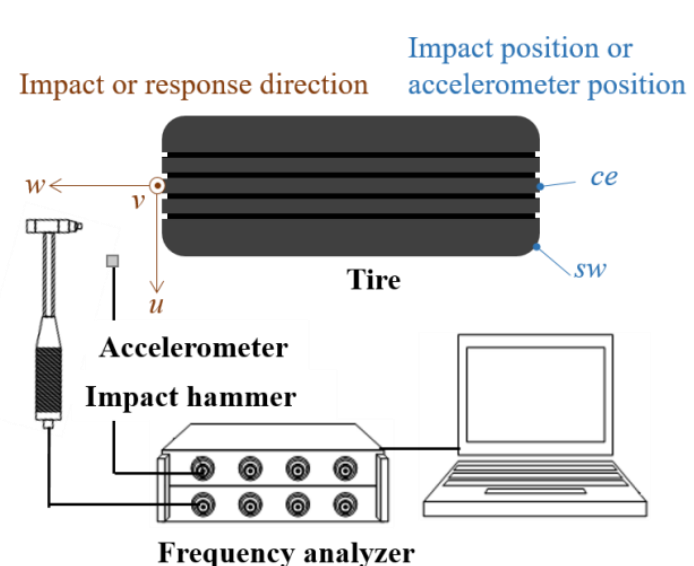

Fig.3 Test setup for experimental modal analysis

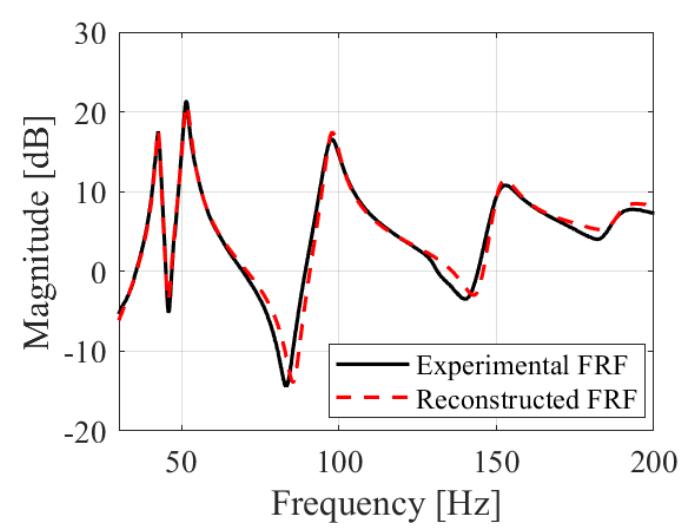

(a) $u$-direction excitation

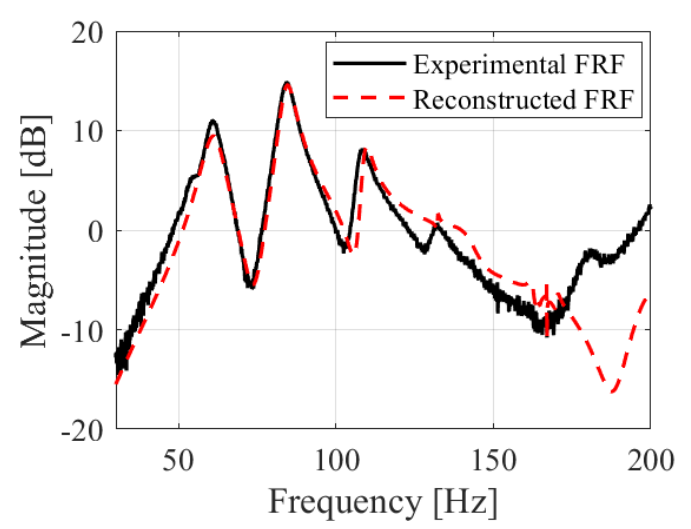

(b) $v$-direction excitation

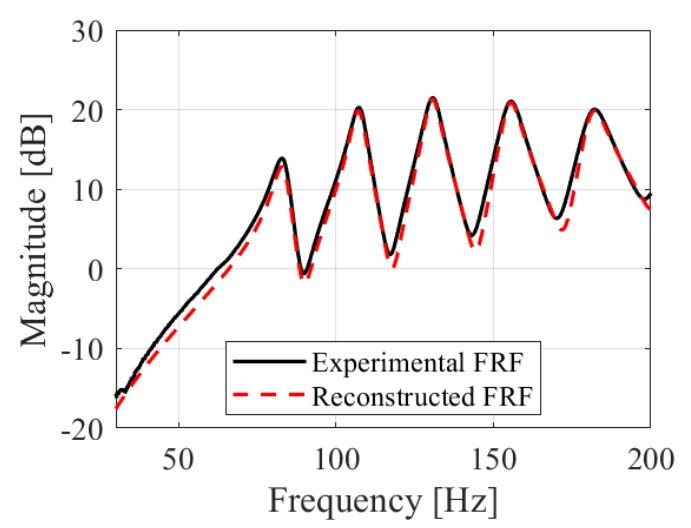

(c) $w$-direction excitation

Fig.4 Comparison between the experimental and reconstructed by EMA results of the magnitude of the accelerance FRFs parts of the $\mathrm{FRF}^{(10)}$. Fig. 4 presents the comparisons between the experimentally obtained and reconstructed FRFs of the input at sw and the response point at ce in the lateral direction, the input and response at ce in the circumferential direction, and the input and response at ce in the radial direction, respectively. The reconstructed FRFs are calculated by Eq. (7). The reconstructed FRFs and the experimental FRFs are in good agreement, and the estimation accuracy is considered to be high.

\subsection{Parameter identification}

Model parameters are obtained by comparison with the modal parameters. The formulas for the natural frequencies for each mode $f_{\text {tra }}, f_{\text {tor }}, f_{\mathrm{r}, n}$, and $f_{\mathrm{b}, m}$ are shown in Eq. (11), representing the natural frequency formulas of the lateral translational, torsional, radial, and lateral bending modes, respectively:

$$
\begin{aligned}
f_{\text {tra }}= & \frac{1}{2 \pi} \sqrt{\frac{K_{y}}{\rho b} \frac{1}{l}}, f_{\text {tor }}=\frac{1}{2 \pi} \sqrt{\frac{K_{\theta}}{\rho b} \frac{1}{l}} \\
f_{\mathrm{r}, n}= & \frac{1}{2 \pi}\left\{\frac{S_{0}}{\rho b} \frac{n^{2}\left(n^{2}-1\right)}{\left(n^{2}+1\right) R^{2}}\right. \\
+ & \left.\frac{K_{\theta}}{\rho b} \frac{1}{\left(n^{2}+1\right) l}+\frac{K_{r}}{\rho b} \frac{n^{2}}{\left(n^{2}+1\right) l}\right\}^{\frac{1}{2}} \\
f_{\mathrm{b}, m}= & \frac{1}{2 \pi}\left[\left\{\frac{S_{0}}{\rho b} \frac{m^{2}\left(m^{2}-1\right) l}{3 R^{2}}+\frac{K_{y}}{\rho b} \frac{R^{2}}{m^{2}} \frac{1}{l}\right.\right. \\
& \left.\left.+\frac{K_{\theta}}{\rho b} l+\frac{K_{r}}{\rho b} m^{2} l\right\} /\left(\frac{R^{2}}{m^{2}}+\frac{l^{2}}{3}+\frac{l^{2}}{3} m^{2}\right)\right]^{\frac{1}{2}}
\end{aligned}
$$

where $\mathrm{m}$ and $\mathrm{n}$ are natural numbers. The above formulas are calculated from, $S_{0} / \rho b, K_{y} / \rho b, K_{\theta} / \rho b$, and $K_{r} / \rho b, R$ and $l$. The relation between the natural frequencies and the stiffness terms can be written as,

$$
\mathbf{f}_{\exp }=\mathbf{C}_{\mathbf{K}} \mathbf{K}_{\mathrm{eq}}
$$

where $\mathbf{f e x p}_{\text {is a }} 2+M+N$ row vector of values calculated from the experimentally obtained natural frequencies. $\mathbf{K}_{\mathbf{e q}}$ is a four row vector of stiffness terms including $S_{0} / \rho b, K_{y} / \rho b, K_{\theta} / \rho b$, and $K_{r}$ $/ \rho b$. $\mathbf{C}_{\mathbf{K}}$ is the $(2+M+N) \times 4$ transfer function matrix and is calculated by $m, n, R$, and $l$.

$$
\begin{aligned}
& \mathbf{f}_{\exp }=\left\{\begin{array}{lllll}
\left(2 \pi f_{t r a}\right)^{2} & \left(2 \pi f_{t r a}\right)^{2} & \left(2 \pi f_{r, 1}\right)^{2} & \ldots & \left(2 \pi f_{r, N}\right)^{2}
\end{array}\right. \\
& \left.\left(2 \pi f_{b, 1}\right)^{2} \quad \cdots \quad\left(2 \pi f_{b, M}\right)^{2}\right\} \\
& \mathbf{K}_{\mathrm{eq}}=\left\{\begin{array}{llll}
\frac{S_{0}}{\rho b} & \frac{K_{y}}{\rho b} & \frac{K_{\theta}}{\rho b} & \frac{K_{r}}{\rho b}
\end{array}\right\}^{T}
\end{aligned}
$$

The modal damping ratios can be obtained from the ratio between the real and imaginary parts of the equivalent stiffness $\mathbf{K}$ in each mode:

$$
\boldsymbol{\eta}_{\text {exp }}=\frac{\operatorname{Im}(\mathbf{K})}{\operatorname{Re}(\mathbf{K})}=\frac{\operatorname{Im}(\mathbf{K} / \rho b)}{\operatorname{Re}(\mathbf{K} / \rho b)}
$$

The relation between the experimentally obtained modal damping ratios and the hysteresis damping ratio is considered and can be written as

$$
\boldsymbol{\eta}_{\exp }=\mathbf{C}_{\boldsymbol{\eta}} \boldsymbol{\eta}_{\text {eq }}
$$

where $\eta_{\exp }$ is a $2+M+N$ row vector of values calculated from the experimentally obtained natural frequencies. $\eta_{\mathbf{e q}}$ is a four row vector of stiffness terms including $S_{0} / \rho b, K_{y} / \rho b, K_{\theta} / \rho b$, and $K_{r}$ $/ \rho b . \mathbf{C}_{\eta}$ is the $(2+M+N) \times 4$ transfer function matrix and is calculated by $m, n, R$, and $l$.

$$
\begin{aligned}
& \boldsymbol{\eta}_{\exp }=\left\{\begin{array}{llllllll}
\eta_{t r a} & \eta_{t o r} & \eta_{r, 1} & \cdots & \eta_{r, N} & \eta_{b, 1} & \cdots & \eta_{b, M}
\end{array}\right\}^{T} \\
& \boldsymbol{\eta}_{\text {eq }}=\left\{\begin{array}{llll}
\eta_{S} & \eta_{y} & \eta_{\theta} & \eta_{r}
\end{array}\right\}^{T}
\end{aligned}
$$


The stiffness term $\mathbf{K}_{\mathbf{e q}}$ and $\eta_{\mathbf{e q}}$ can be obtained by the inverse matrix method. $\mathbf{C}_{\mathbf{K}}$ and $\mathbf{C}_{\boldsymbol{\eta}}$ is not usually a regular matrix. Thus, the pseudo-inverse matrix is used instead of the inverse matrix.

$$
\mathbf{K}_{\text {eq }}=\mathbf{C}_{\mathbf{K}}{ }^{+} \mathbf{f}_{\text {exp }}
$$$$
\boldsymbol{\eta}_{\text {eq }}=\mathbf{C}_{\boldsymbol{\eta}}^{+} \boldsymbol{\eta}_{\exp }
$$

Then, mass term $\rho b$ is derived by the following equation.

$$
\rho b=\frac{\operatorname{Re}\left(\frac{\rho b}{K_{t r a}}+\frac{\rho b}{K_{t o r}}+\sum_{n=1}^{N} \frac{\rho b}{K_{r, n}} n^{2}+\sum_{m=1}^{M} \frac{\rho b}{K_{b, m}} \frac{R^{2}}{m^{2}}\right)}{R_{t r a}+R_{t o r}+\sum_{n=1}^{N} R_{r, n}+\sum_{p=1}^{m} R_{b, m}}
$$

The model parameters can be determined from Eqs.(17) given $R$ and $l$. The numerator and denominator of Eq.(18) can be calculated by $\mathbf{K}_{\mathbf{e q}}$ and the residual term from the EMA, respectively. The following equations are considered to be a function to assess the identification accuracy $J_{1}$ and $J_{2}$ as the root mean square value of the relative errors between the EMA and recalculated by the model parameters.

$$
\begin{aligned}
J_{1}(R, l)=\frac{1}{\sqrt{3 n}} & {\left[\sum_{p=1}^{2+m+n}\left\{\frac{\left(f_{p}\right)_{\exp }-\left(f_{p}\right)_{\text {ana }}}{\left(f_{p}\right)_{\exp }} \times 100\right\}^{2}\right.} \\
& +\sum_{p=1}^{2+m+n}\left\{\frac{\left(\eta_{p}\right)_{\exp }-\left(\eta_{p}\right)_{\text {ana }}}{\left(\eta_{p}\right)_{\exp }} \times 100\right\}^{2} \\
& \left.+\sum_{p=1}^{2+m+n}\left\{\frac{\left(R_{p}\right)_{\exp }-\left(R_{p}\right)_{a n a}}{\left(R_{p}\right)_{\exp }} \times 100\right\}^{2}\right]^{\frac{1}{2}} \\
J_{2}(R, l)=\frac{1}{\sqrt{2 n}} & {\left[\sum_{p=1}^{2+m+n}\left\{\frac{\left(f_{p}\right)_{\exp }-\left(f_{p}\right)_{\text {ana }}}{\left(f_{p}\right)_{\exp }} \times 100\right\}^{2}\right.} \\
+ & \left.\sum_{p=1}^{2+m+n}\left\{\frac{\left(\eta_{p}\right)_{\exp }-\left(\eta_{p}\right)_{\text {ana }}}{\left(\eta_{p}\right)_{\exp }} \times 100\right\}^{2}\right]^{\frac{1}{2}}
\end{aligned}
$$

where the subscripts exp and ana denote the modal parameter estimated by EMA and the modal parameters recalculated from the model parameters. The values of $R$ and $l$ are defined as the set of parameters leading to minimum $J_{1}$ or $J_{2}$. Fig. 5 visually presents the assessment function $J_{1}$ and $J_{2}$. The range of $\mathrm{R}$ and 1 are defined as the radius of the maximum outer diameter $\pm 10 \%$ and half of the tire width $\pm 50 \%$. The red circle in Fig. 5 is the point at which $R$ and $l$ are optimized. In the case of $J_{1}$, the optimization point is on the boundary of the analytical range. As this parameter greatly deviates from the actual tire size, it cannot be adopted. On the other hand, in the case of $J_{1}$, the optimization point is within the analytical range. The value of $J_{1}$ is higher than that of $J_{2}$ because of the error in the residual terms. If we use $J_{1}$, the reproducibility of the peak may decrease. Thus, $J_{2}$ is adopted as the function for assessing the identification accuracy. The point in Fig.5(b) when $R=0.3260$ and $l=0.0750$ is the set of $R$ and $l$ leading to minimum $J_{2}$. Table 1 summarizes the identified modal parameters. The tension of tread ring $S_{0}$, and sidewall stiffness $K_{y}$, $K_{\theta}$, and $K_{r}$ are calculated by multiplying $\mathbf{K}_{\text {eq }}$ and $\rho b$. Fig. 6 shows comparisons between the measured FRFs and recalculated FRFs using model parameters and Eqs.(8)-(10) under the input at sw and the response at ce in the lateral direction, the input and response at ce in the circumferential direction, and the input and response at ce in the radial direction, respectively. The FRFs show good correlation for the three conditions when the experimental and recalculated results are compared.

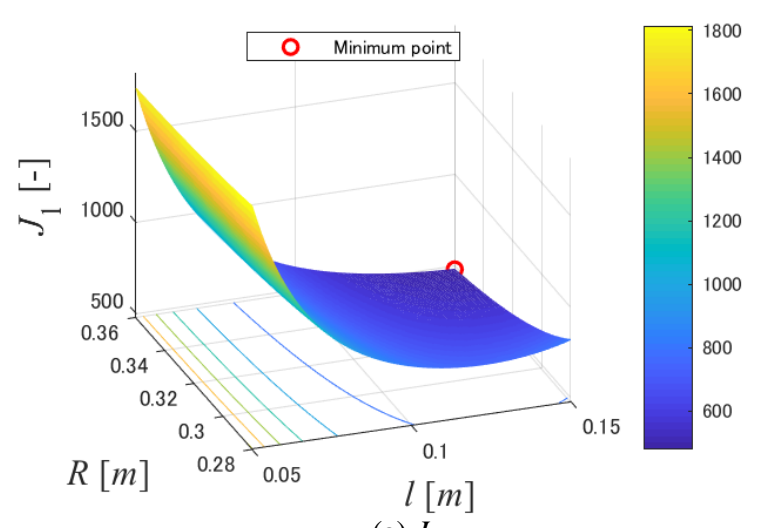

(a) $J_{1}$

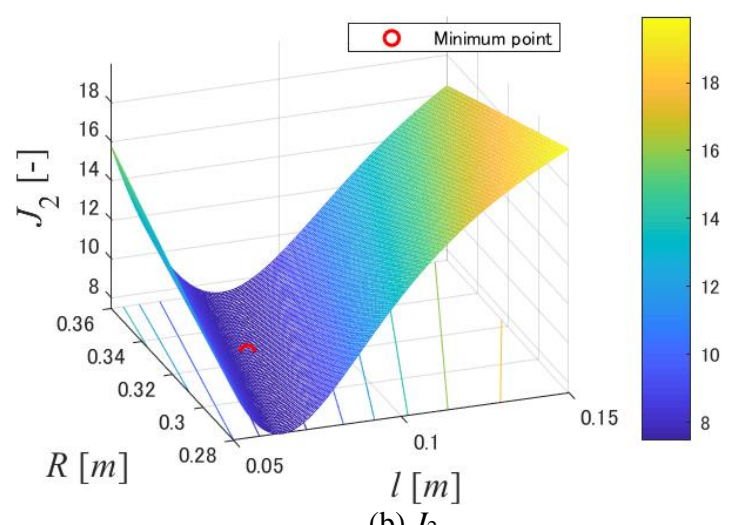

(b) $J_{2}$

Fig.5 Assessment function

Table 1 Model parameters

\begin{tabular}{c|c|c}
\hline $\begin{array}{c}\text { Model } \\
\text { parameter [unit] }\end{array}$ & Description & Numerical value \\
\hline \hline$\rho b[\mathrm{~kg} / \mathrm{m}]$ & Linear density & 12.71 \\
\hline$R[\mathrm{~m}]$ & Tread radius & 0.3260 \\
\hline$l[\mathrm{~m}]$ & Tread half width & 0.0750 \\
\hline$S_{0}[\mathrm{~N} / \mathrm{m}]$ & $\begin{array}{c}\text { Tension due to inflation } \\
\text { pressure }\end{array}$ & $4.191 \times 10^{3}$ \\
\hline$K_{y}\left[\mathrm{~N} / \mathrm{m}^{2}\right]$ & Lateral sidewall stiffness & $6.086 \times 10^{3}$ \\
\hline$K_{\theta}\left[\mathrm{N} / \mathrm{m}^{2}\right]$ & $\begin{array}{c}\text { Circumferential sidewall } \\
\text { stiffness }\end{array}$ & $1.129 \times 10^{4}$ \\
\hline$K_{r}\left[\mathrm{~N} / \mathrm{m}^{2}\right]$ & radial sidewall stiffness & $2.997 \times 10^{4}$ \\
\hline$\eta_{s}[-]$ & $\begin{array}{c}\text { Structural damping } \\
\text { coefficient related to } S_{0}\end{array}$ & 0.0399 \\
\hline$\eta_{y}[-]$ & $\begin{array}{c}\text { Structural damping } \\
\text { coefficient related to } K_{y}\end{array}$ & 0.0317 \\
\hline$\eta_{\theta}[-]$ & $\begin{array}{c}\text { Structural damping } \\
\text { coefficient related to } K_{\theta}\end{array}$ & 0.1215 \\
\hline$\eta_{r}[-]$ & $\begin{array}{c}\text { Structural damping } \\
\text { coefficient related to } K_{r}\end{array}$ & 0.0440 \\
\hline \hline
\end{tabular}




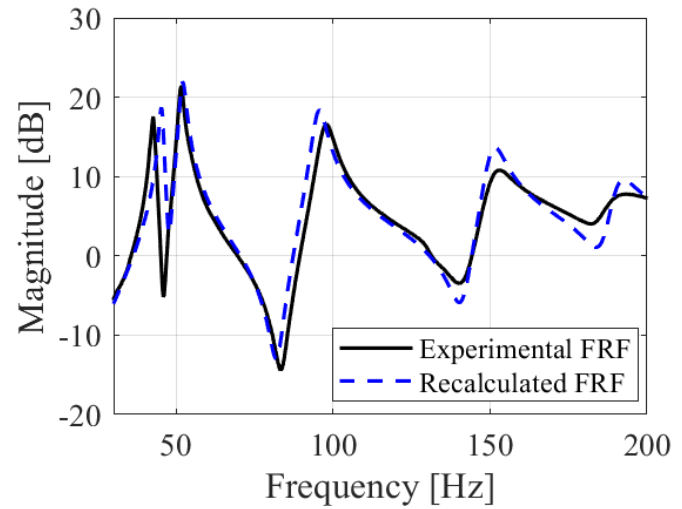

(a) $u$-direction excitation

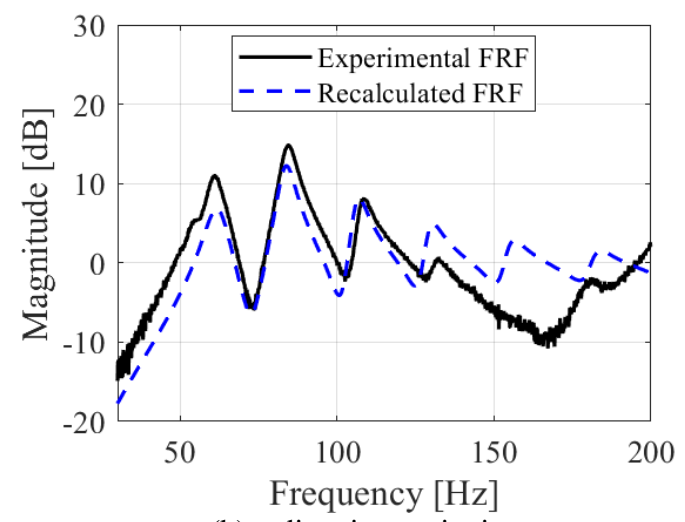

(b) $v$-direction excitation

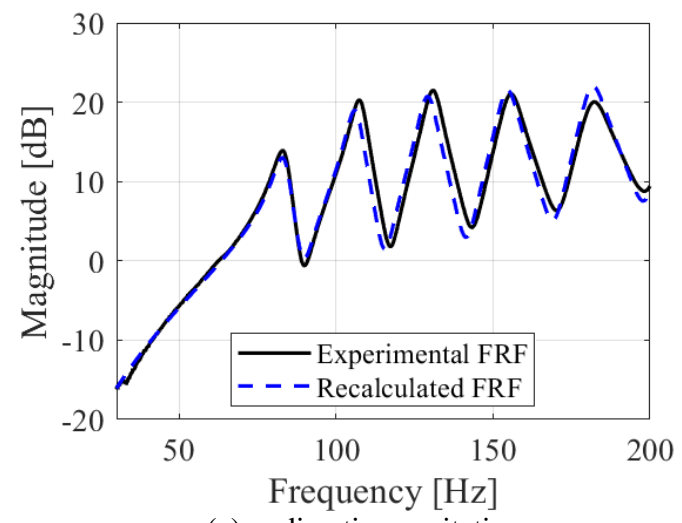

(c) $w$-direction excitation

Fig.6 Comparison between the experimental FRFs and the recalculated FRFs based on model parameters.

\section{Conclusion}

In this research, we proposed simplified model parameter estimation for a three-dimensional flexible ring-based model. This method enables the identification of modal parameters by using EMA and the calculation of model parameters by comparing them with the modal parameters calculated from the model. The method we used for EMA is based on a method that involves linear fit of the frequency response function and we confirmed that the modal parameters can be estimated with high accuracy. The use of the aforementioned method made it possible to identify the model parameters by using not only the natural frequency but also the structural damping coefficient and the residual term. The dimensional parameters were determined to be the tread radius $R$ and tread half width $l$, and the values of $R$ and $l$ were defined as the set of parameters leading to the minimum assessment function.
Among the modal parameters, the error of the residual term becomes larger due to the influence of the error of the nonlinear terms, which are the natural frequency and the structural damping coefficient. Thus, the root mean square value of the relative errors between the estimated and recalculated modal parameters (except for the residual term of the model parameters) is adopted as the function to assess the identification accuracy. The recalculated results showed good correlation with the experimental results. This method simplifies parameter optimization, and has high FRF reproducibility. The advantage of the proposed identification method is that it can proceed with only vibration test data without requiring the tire geometry or material parameter details.

\section{References}

(1) H. Pacejka: Tire and Vehicle dynamics $3^{\text {rd }}$ edition, Butterworth-Heinemann, pp.582-586 (2012).

(2) Katsunori Tanaka, Ichiro Kageyama: Study of Tire Model for the Estimation of Design Parameters, Transactions of Society of Automotive Engineers of Japan (in Japanese), Vol.30, No,2, pp.99-104 (1999).

(3) M. Matsubara, N. Tsujiuchi, T. Koizumi, A. Ito and K. Bito: Natural frequency analysis of tire vibration using a thin cylindrical shell model, SAE technical paper, 2015-01-2198 (2015).

(4) M. Matsubara, D. Tajiri, T. Ise and S.Kawamura: Vibrational response analysis of tires using a three-dimensional flexible ring-based model, Journal of Sound and Vibration, Vol.408, pp.368-382 (2017).

(5) Y. J. Kim, J. S. Bolton: Effect of rotation on the dynamics of a circular cylindrical shell with application to tire vibration, Journal of Sound and Vibration, Vol.304, pp.605-621 (2004)

(6) I. Lopez, R. E. A. Blom, N. B. Roozen, H.Nijmeijer: Modelling vibrations on deformed rolling tyres - a modal approach, Journal of Sound and Vibration, Vol.307, pp.481494 (2007).

(7) I. F. Kozhevnikov: Vibrations of a rolling tyre, Journal of Sound and Vibration, Vol.331, pp.1669-1685 (2012).

(8) M. Matsubara, M. Horiuchi, T. Ise and S.Kawamura: Simplified modelling and eigenvalue analysis of coupling system between tire and wheel, Transactions of the JSME (in Japanese), Vol.82, No.844, Paper No.16-00300, p.1 - 18 (2016).

(9) D. Tajiri, M. Matsubara, T. Ise, S. Kawamura: Evaluation of tire basic stiffness using a low degree of freedom dynamic model and vibration tests, 2018 JSAE Annual Congress Proceedings (in Japanese), No.89-16, pp.2224-2228 (2016).

(10)M. Matsubara, T. Ise and S.Kawamura: Application of modal properties identification to multi-degree-of-freedom system using simultaneous equations of the real and imaginary parts of frequency response function, Transactions of the JSME (in Japanese), Vol.84, No.860, Paper No.17-00540, pp.1 - 21 (2018).

(11)P. Kindt, P. Sas and W. Desmet: Development and validation of a three-dimensonal ring-based structural tyre model, Journal of Sound and Vibration, Vol.326, pp.852-869 (2009).

(12)S. Timoshenko, S.W. Kriger: Theory of plates and shells, McGraw-Hill(in Japanese), pp.474-476 (1980).

(13)C.G. Cooley, R.G. Parker: Limitation of an inextensible model for the vibration of high-speed rotating elastic rings with attached space-fixed discrete stiffness, European Journal of Mechanics / A Solids, Vol.54, pp.187-197 (2015).

(14) B. Peeters, H. der Auweraer, P. Guillaume, J. Leuridan: The PolyMAX frequency-domain method: a new standard for modal parameter estimation?, Shock and Vibration, Vol.11, pp.395-409 (2004). 